\title{
Práticas do cuidado com recém-nascido em tempos de pandemia: revisão integrativa
}

\author{
Practices of care with newborn in pandemic times: integrative review \\ Prácticas de atención al recién nacido en tiempos de pandémica: revisión integrativa
}

Recebido: 01/02/2021 | Revisado: 08/02/2021 | Aceito: 13/02/2021 | Publicado: 21/02/2021

Cláudia Martins da Costa

ORCID: https://orcid.org/0000-0001-5904-6829 Universidade do Estado de Minas Gerais, Brasil E-mail: claudiacostamello.92@gmail.com

Thays Cristina Pereira Barbosa ORCID: https://orcid.org/0000-0003-1816-0662 Universidade do Estado de Minas Gerais, Brasil E-mail: thayscristina19@gmail.com

Caique Alves Rezende

ORCID: https://orcid.org/0000-0003-0956-7321 Universidade do Estado de Minas Gerais, Brasil E-mail: caiquerezende25@gmail.com

Letícia Blandim Fonseca

ORCID: https://orcid.org/0000-0002-8110-9301 Universidade do Estado de Minas Gerais, Brasil

E-mail: leticiablandimfonseca@gmail.com

Jhenifer Alves de Araújo

ORCID: https://orcid.org/0000-0002-7777-8318 Universidade do Estado de Minas Gerais, Brasil E-mail: jheni.araujo1998@gmail.com Laeny Catarina Dias Freitas ORCID: https://orcid.org/0000-0002-9387-4334 Universidade do Estado de Minas Gerais, Brasil E-mail: laenycatarina@gmail.com

Regina Consolação dos Santos ORCID: https://orcid.org/0000-0002-7393-3210 Universidade do Estado de Minas Gerais, Brasil E-mail: regina.consolação@uemg.br

\begin{abstract}
Resumo
O objetivo deste estudo foi identificar evidências científicas sobre boas práticas no cuidado ao recém-nascido, da sala de parto ao domicílio, em tempos de COVID-19. Trata-se de uma revisão Integrativa. Assim, o delineamento desse estudo surgiu a partir da seguinte questão de pesquisa: o que se sabe até o momento da literatura científica sobre as práticas do cuidado ao recém-nascido, em tempos de pandemia? Após percorrer as etapas metodológicas foi possível identificar 05 artigos, todas as publicações são datadas no ano 2020 e focadas na prevenção e os cuidados prestados aos recém-nascidos. Diante da analise da literatura disponível, evidenciou-se diversas divergências sobre a implementação das praticas de cuidado. Visto isso, torna-se viável a criação de um protocolo de assistência aos recém-nascidos em tempos de pandemia de COVID-19, a fim de estabelecer um padrão de qualidade. Ainda sim, é preciso a maior realização de pesquisas referentes à assistência aos recém-nascidos considerando o período de pandemia do COVID-19, em especial de autores brasileiros.
\end{abstract}

Palavras-chave: Recém-nascido; Cuidados de enfermagem; Pandemias; Infecções por Coronavírus; Covid-19.

\begin{abstract}
The aim of this study was to identify scientific evidence about good practices in newborn care, from the delivery room to the home, in times of COVID-19. This is an Integrative review. Thus, the design of this study arose from the following research question: what is known so far in the scientific literature on newborn care practices, in times of pandemic? After going through the methodological steps, it was possible to identify 06 articles, all publications are dated in 2020 and focused on prevention and care provided to newborns. In view of the analysis of the available literature, there were several differences on the implementation of care practices. In view of this, it is feasible to create a protocol to assist newborns in times of pandemic of COVID-19, in order to establish a quality standard. Still, more research is needed regarding assistance to newborns considering the COVID-19 pandemic period, especially of Brazilian authors.
\end{abstract}

Keywords: Newborn; Nursing care; Pandemics; Coronavirus infections; Covid-19. 


\section{Resumen}

El objetivo de este estudio fue identificar evidencia científica sobre buenas prácticas en el cuidado del recién nacido, desde la sala de partos hasta el hogar, en tiempos de COVID-19. Esta es una revisión integradora. Así, el diseño de este estudio surgió a partir de la siguiente pregunta de investigación: ¿qué se conoce hasta ahora en la literatura científica sobre las prácticas de cuidado del recién nacido, en tiempos de pandemia? Luego de pasar por los pasos metodológicos, fue posible identificar 06 artículos, todas las publicaciones están fechadas en 2020 y enfocadas a la prevención y atención brindada a los recién nacidos. A la vista del análisis de la literatura disponible, hubo varias diferencias en la implementación de las prácticas asistenciales. Ante esto, es factible crear un protocolo de asistencia a los recién nacidos en tiempos de pandemia de COVID-19, con el fin de establecer un estándar de calidad. Aún así, se necesita más investigación sobre la asistencia a los recién nacidos considerando el período de la pandemia de COVID19, especialmente de autores brasileños.

Palabras clave: Recién nacido; Cuidado de enfermera; Pandemias; Infecciones por coronavirus; Covid-19.

\section{Introdução}

Em dezembro do ano de 2019 ocorreu a transmissão de um novo coronavírus (SARS-CoV-2), identificado primeiro em Wuhan na China e rapidamente se disseminou entre as pessoas. A doença causada pelo Covid-19 provoca sintomas que podem variar entre uma assintomatologia, um resfriado, pneumonia, síndrome respiratória grave, lesões cardíacas e até mesmo a morte. No ano de 2020, este vírus causou uma pandemia, se tornando um grave problema de saúde global que paralisou o mundo frente a sua virulência, patogenicidade e capacidade de infecção (Brasil, 2020; Rose, et al., 2020).

Com sua alta capacidade de disseminação e a falta de informações sobre o novo Covid-19, fez-se necessário à implementação de medidas de contenção como: o uso de máscaras, isolamento social, o reforço da higienização das mãos e o uso do álcool 70\%. Além destas medidas, os serviços de cuidados em saúde precisaram se adaptar às novas medidas de prevenção constantes tendo assim novos desafios em exercer o cuidado (OMS, 2020).

A pandemia por Covid-19 levou a interrupção de diversos serviços de saúde a fim de conter a infecção. Neste período os pais de crianças menores de um ano também evitaram levar os filhos aos locais de maior probabilidade de infecção. (OPAS, 2020; Brasil, 2020). As crianças menores de 15 anos têm mostrado menos susceptibilidade às formas mais graves, sendo que poucas crianças vão apresentar sintomas, e as razões para isso permanecem obscuras. No entanto, não se é conhecido ainda, as reações do corona-vírus em recém-nascidos (Rose et al., 2020).

Após o parto, os recém-nascidos merecem um atendimento especial devido transformações fisiológicas que acontecem inclusive nas trocas gasosas e padrão cardiovascular. Os cuidados prestados ao neonato irão favorecer sua adaptação para a vida extrauterina e poderão determinar sua saúde imediata até a vida adulta, além de estar relacionado à redução da mortalidade infantil. Neste momento é realizado um conjunto de condutas que juntamente com um cuidado humanístico e integral, poderão proporcionar qualidade de vida aquela família (Ledo, et al., 2021).

Sabe se que, recém-nascidos tem maior risco de morte mesmo antes do surgimento do novo corona-vírus e necessitam cuidados constantes, e por vezes específicos principalmente aqueles que nascem com alguma comorbidade. Além disso, o sistema imunológico dos neonatos é ainda imaturo para lidar com eficiência contra o vírus, sendo assim, é necessário prevenir a contaminação uma vez que não se conhece inteiramente a nova doença e não existe um tratamento seguro e eficaz (Dilli \& Tasoglu, 2020; OPAS, 2020).

Desse modo, a avaliação do índice de Apgar, o exame físico, o contato pele a pele com a mãe, a amamentação na primeira hora de vida, são ações que devem ser realizadas, mas com uma nova perspectiva, tomando todos os cuidados para não contaminação do neonato, preservando sua integridade (Sociedade Brasileira de Pediatria, 2020).

Diante da dificuldade deste momento, este trabalho justifica-se pela necessidade de conhecer as medidas de contenção ao novo Corona-vírus nas práticas do cuidado ao recém-nascido e pela falta de estudos da nova situação de saúde. O presente estudo tem como objetivo identificar evidências científicas sobre boas práticas no cuidado ao recém-nascido, da sala de parto ao domicílio, em tempos de COVID-19. 


\section{Metodologia}

Trata-se de um estudo de Revisão Integrativa (RI), que tem como objetivo sintetizar os resultados obtidos em pesquisas sobre um tema ou questão de maneira sistemática, ordenada e abrangente, fornecendo informações mais amplas sobre o assunto e o problema a ser pesquisado, proporcionando assim ao pesquisador um corpo de conhecimento maior (Ercole, Melo \& Alcoforado 2014). Através dos resultados encontrados, foi realizada uma análise qualitativa, por meio de abordagem exploratória e interpretação dos dados coletados nas bases (Pereira, Shitsuka, Parreira \& Shitsuka, 2018).

Assim, o delineamento desse estudo surgiu a partir da seguinte questão de pesquisa: o que se sabe até o momento da literatura científica sobre as práticas do cuidado ao recém-nascido, em tempos de pandemia?

O estudo foi realizado a partir da consulta de artigos científicos disponíveis nas bases de dados nacionais e internacionais como a Base de dados de Enfermagem (BDENF), Scientific Eletronic Library Online (SCIELO), National Library of Medicine National Institutes of Health (PUBMED), Medical Literature Analysis and Retrieval Sistem on-line (MEDLINE) e Literatura Latino-Americana e do Caribe em Ciências da Saúde (LILACS).

A estratégia de busca adotada foi à utilização de descritores e operadores booleanos "OR" e "AND" a fim de aumentar o escopo da busca dos artigos. Na descrição dos descritores foi utilizado o acrômio PICOT, ao qual teve como intuito fortalecer a busca dos artigos nas bases de dados. Foram incluídos descritores e seus termos alternativos, válidos nos Descritores em Ciência da Saúde (DeCS), sendo eles: Recém-Nascido OR Criança Recém-Nascida OR Crianças RecémNascidas OR Lactente Recém-Nascido OR Lactentes Recém-Nascidos OR Neonato OR Neonatos OR Recém-Nascido (RN) OR Recém-Nascidos AND Cuidados de Enfermagem OR Assistência de Enfermagem OR Atendimento de Enfermagem OR Cuidado de Enfermagem AND Pandemias OR Pandemia AND Infecções por Coronavirus OR COVID-19.

A busca foi realizada em dezembro de 2020 e os critérios de inclusão serão: artigos completos, disponíveis na integra, gratuitos, teses, dissertações, artigos publicados no período de 2015 a 2020 e artigos na língua portuguesa, inglesa e espanhola. Foram excluídos todos aqueles estudos que não contemplaram o tema a ser abordado. A seleção dos artigos foi realizada após a leitura criteriosa dos títulos e resumos para exclusão de publicações duplicadas e daquelas que não atenderem os propósitos da pesquisa. Os artigos selecionados foram lidos na íntegra para extração das variáveis de interesse: Recém-nascido, parto, puerpério, pandemia, COVID-19, prevenção e cuidados ao recém-nascido. A seleção dos estudos foi realizada por dois pesquisadores, em buscas independentes. Em caso de desacordo, um terceiro autor foi consultado e a decisão final foi tomada por consenso.

Foi elaborada uma matriz de síntese com os artigos selecionados, contendo as informações: título da obra, autoria, revista e ano de publicação. Os dados foram separados de acordo com o seu nível de evidência.

\section{Resultados}

Após percorrer as etapas metodológicas estabelecidas na revisão integrativa, identificou-se 15 artigos, foram incluídos 5 artigos mediante os critérios estabelecidos. Em seguida, foram excluídos 10 artigos por não atenderem aos critérios de inclusão. Todos os estudos foram publicados em inglês, apesar das diferentes nacionalidades dos autores envolvidos. As etapas do processo metodológico foram descritas em um fluxograma (Figura 1). 
Research, Society and Development, v. 10, n. 2, e40410212552, 2021

(CC BY 4.0) | ISSN 2525-3409 | DOI: htttp://dx.doi.org/10.33448/rsd-v10i2.12552

Figura 1. Etapas do processo metodológico utilizadas para realização do estudo.

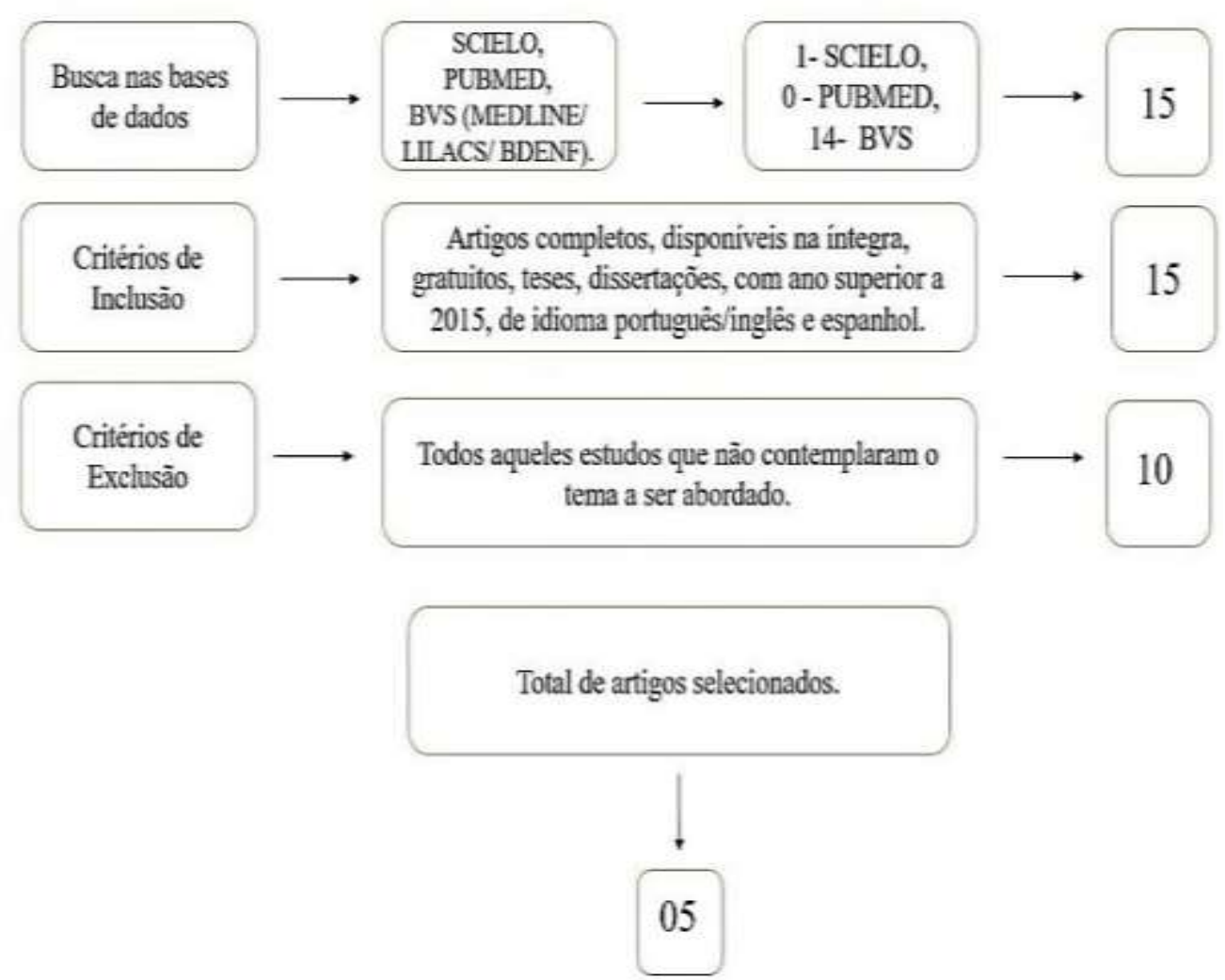

Fonte: Acervo pessoal dos autores (2020).

Devido à atualidade do tema do tema abordado, todas as publicações são datadas no ano 2020, sendo que os artigos incluídos neste estudo empregaram o seguinte delineamento metodológico: dois estudos de revisão de escopo, um estudo de revisão integrativa, dois artigos de opiniões de especialistas (editoriais, consensos). Evidencia-se as literaturas usadas para criação deste estudo (Quadro 1). 
Quadro 1. - Distribuição dos artigos utilizados conforme detalhamento quantitativo. Título da obra, autoria, revista e ano.

\begin{tabular}{|c|c|c|c|}
\hline Título da Obra & Autoria & Revista & Ano \\
\hline $\begin{array}{l}\text { Recomendações assistenciais à } \\
\text { parturiente, puérpera e recém- } \\
\text { nascido durante a pandemia de } \\
\text { COVID-19: revisão de escopo }\end{array}$ & $\begin{array}{l}\text { Mascarenhas, Victor Hugo Alves, Caroci- } \\
\text { Becker, Adriana, Venâncio, Kelly Cristina } \\
\text { Máxima Pereira, Baraldi, Nayara Girardi, } \\
\text { Durkin, Adelaide Caroci, \& Riesco, Maria } \\
\text { Luiza Gonzalez. }\end{array}$ & $\begin{array}{l}\text { Revista Latino-Americana De } \\
\text { Enfermagem }\end{array}$ & 2020 \\
\hline $\begin{array}{l}\text { Best practices in newborn care } \\
\text { in COVID-19 times: an } \\
\text { integrative review }\end{array}$ & $\begin{array}{l}\text { Góes, Fernanda Garcia Bezerra; Santos, } \\
\text { Andressa Silva Torres dos; Lucchese, } \\
\text { Ingrid; Silva, Laura Johanson da; Silva, } \\
\text { Liliane Faria da; Silva, Maria da } \\
\text { Anunciação. }\end{array}$ & Texto e Contexto Enfermagem & 2020 \\
\hline $\begin{array}{l}\text { Prevention and control } \\
\text { measures for neonatal COVID- } \\
19 \text { infection: a scoping review }\end{array}$ & $\begin{array}{l}\text { Freitas, Bruna Hinnah Borges Martins de; } \\
\text { Alves, Mayrene Dias de Sousa Moreira; } \\
\text { Gaíva, Maria Aparecida Munhoz. }\end{array}$ & $\begin{array}{l}\text { Revista Brasileira de } \\
\text { Enfermagem }\end{array}$ & 2020 \\
\hline $\begin{array}{l}\text { Neonatal COVID-19: little } \\
\text { evidence and the need for more } \\
\text { information }\end{array}$ & $\begin{array}{l}\text { Procianoy, Renato Soibelmann; Silveira, } \\
\text { Rita C; Manzoni, Paolo; Sant'Anna, } \\
\text { Guilherme. }\end{array}$ & Jornal de Pediatria & 2020 \\
\hline $\begin{array}{l}\text { Benefits and Risks of Visitor } \\
\text { Restrictions for Hospitalized } \\
\text { Children During the COVID } \\
\text { Pandemic. }\end{array}$ & $\begin{array}{l}\text { Virani, Alice K; Puls, Henry T; Mitsos, } \\
\text { Rebecca; Longstaff, Holly; Goldman, Ran } \\
\text { D; Lantos, John D. }\end{array}$ & Pedriatrics & 2020 \\
\hline
\end{tabular}

Fonte: Acervo pessoal dos autores (2020).

A partir da análise dos artigos, foi possível identificar grande parte dos estudos abordaram o surto de COVID-19 em recém-nascidos e crianças, focando principalmente na prevenção e os cuidados prestados a esta referida população desde o parto até o puerpério (Quadro 1).

\section{Discussão}

Mediantes os resultados encontrados, foi possível identificar que as pesquisas referentes aos cuidados com os recémnascidos neste tempo de pandemia, ainda precisam de mais estudos, tendo em vista que, o Covid-19 é uma temática extremamente nova para os pesquisadores e profissionais que estão atuando diretamente nos cuidados da referida população. Destaca-se também que os recém-nascidos e crianças são grupos de risco por apresentarem um sistema imunológico ainda imaturo, estando vulneráveis a esta doença e desta forma, requerendo mais cuidados tanto por parte dos cuidadores quanto dos profissionais.

Segundo Góes, et al (2020), algumas medidas podem ser tomadas para a proteção dos recém-nascidos com utilização de incubadoras, barreiras físicas entre a mãe e o bebê, nas unidades de terapia intensiva neonatal, estimulando o contato pele a pele, sendo a mãe assintomática e que não possua contato com familiares com síndrome gripal ou infecção respiratória confirmada por SARS-CoV-2, nos últimos 14 dias. Conforme Freitas, Alves \& Gaíva (2020), o contato pele a pele não é recomendado para o controle de Covid-19, embora o contato pele a pele mãe e recém-nascido seja preconizado pelo Ministério da Saúde brasileiro, por ser extremamente benéfico nas primeiras horas de vida.

Divergências entre a admissão de neonatos suspeitos ou com confirmação para o novo Coronavírus nas unidades de terapia intensiva neonatal ou o isolamento e monitoramento dos mesmos, são frequentes. Tanto a equipe quanto a própria unidade de terapia intensiva, devem realizar a preparação de recursos humanos específicos para lidarem com esta determinada população, com equipamentos de tratamento e diagnóstico individualizados, evitando-se que outros pacientes sejam contaminados. Mesmo em outros setores, é esperado que os locais de isolamentos contenham sistemas de ciclo de ar isolado, com pressão negativa (Freitas, Alves \& Gaíva, 2020). 
Os neonatos requerem maior cuidado devido ao sistema imunológico imaturo e a probabilidade de transmissão de mãe para o bebê (Li, Feng \& Shi, 2020). Segundo Wang, et al (2020), é indispensável o monitoramento de perto dos sinais vitais, sintomas respiratórios e sintomas gastrointestinais. A mudança de temperatura do bebê infectado pode apresentar elevada, deprimida ou normal. Nos sintomas cardiovasculares e respiratórios, podem originar respiração difícil, apneia, taquipnéia, taquicardia.

A amamentação também gerou controvérsias em grande parte dos estudos. Evidências mostraram que o aleitamento materno pode facilitar a contaminação de recém-nascidos por gotículas respiratórias durante a amamentação, embora o leite materno seja considerado a principal fonte de nutrição do neonato. Para minimizar esse risco, recomenda-se o uso de máscara facial cirúrgica durante a amamentação, caso ocorra espirro ou tosse, além da higienização correta e contínua das mãos. No Brasil, ainda se recomenda a amamentação mesmo em casos de infecção pela SARS-CoV-2, desde que a mãe tenha o interesse em amamentar e em condições clínicas favoráveis (Freitas, Alves \& Gaíva, 2020; Mascarenhas, et al., 2020).

Devido à pandemia de Covid-19 e seu aumento de transmissões em ambiente de saúde, tornou-se uma ameaça progressivamente cada vez mais constatada em pacientes e profissionais de saúde (Banach, et al., 2015). Grande parte dos hospitais, estão executando medidas de restrições ao número de pessoas que podem visitar os pacientes, o uso dessa estratégia é eficaz e reduz a transmissão da doença, que tem como desafio de equilibrar os danos de diminuir as visitas como forma de proteção aos pacientes e profissionais da saúde (Virani, et al., 2020).

A falta de consenso entre as literaturas sobre quais serão as medidas adotadas em recém-nascidos com suspeita ou confirmação de Covid-19 ou até mesmo assintomáticas, pode dificultar na prestação do cuidado pela equipe multiprofissional que está em contato direto com estes pacientes, o que pode impactar na qualidade da assistência prestada. A enfermagem é primordial no cuidado aos recém-nascidos, por estar prestando assistência direta a estes pacientes, o acompanhamento dos sinais vitais, sintomas respiratórios e gastrointestinais são essenciais para o tratamento dos mesmos que são totalmente dependentes e por apresentarem sinais e sintomas inespecíficos (Freitas, Alves \& Gaíva, 2020).

Embora Mascarenhas, et al (2020), afirme em seu estudo que a COVID-19 seja menos comum em recém-nascidos e crianças menores que três meses, elas possuem um risco elevado para infecção com maior gravidade. Além disso, após o parto os recém-nascidos apresentaram peso, estatura e índices de Apgar adequados, sem nenhum relato de asfixia ou óbito neonatal. Por meio dos estudos analisados, percebe-se que alguns casos de COVID-19 positivos, foram relatados no Brasil por meio do noticiário ou de apresentações clínicas (Procianoy, et al., 2020), o que reforça que a vigilância deve ser mantida nesta população.

Este estudo apresenta limitações. Por se tratar de uma análise por meio de estudos científicos, nota-se que a ocorrência de casos tanto suspeitos quanto confirmados em recém-nascidos ainda são pouco estudados bem como os sinais e sintomas da doença nos mesmos, o que dificulta na determinação do perfil dos pacientes que apresentam a doença no país e nas formas de intervenções adequadas para a diminuição de complicações e contaminações.

\section{Conclusão}

Mediantes a análise dos estudos encontrados, conclui-se que é preciso incentivar a realização de pesquisas referentes à assistência aos recém-nascidos considerando o período de pandemia do COVID-19, em especial de autores brasileiros. Ainda assim, uma melhor analise da produção cientifica sobre os modelos de práticas dos profissionais de saúde dentro deste cenário.

Defronte da leitura completa da literatura disponível, evidenciou-se diversas divergências sobre a implementação das práticas de cuidado. Essas discordâncias podem causar erros de assistência, dificuldades dos profissionais de saúde para apropriar-se das técnicas corretas, possíveis discussões da equipe responsável pelos cuidados dos recém-nascidos, entre outros. Visto isso, torna-se viável a criação de um protocolo de assistência aos recém-nascidos em tempos de pandemia de COVID-19, 
a fim de estabelecer um padrão de qualidade aos atendimentos, uma vez que, os cuidados terão uma base cientifica e comprovada para uma assistência de excelência.

Diante o conteúdo apresentado nesse trabalho, foi dado apenas o primeiro passo com a demonstração das discordâncias dentro da assistência do recém-nascido. A criação de um protocolo de assistência e a escrita de outros artigos futuramente dentro de uma perspectiva constante de pesquisa trará um processo fundamental e necessário para modelar e compreender as necessidades de uma assistência integralizada moldada em uma base cientifica.

\section{Referências}

Banach, D. B., Bearman, G. M., Morgan, D. J., \& Munoz-Price, L. S. (2015). Infection control precautions for visitors to healthcare facilities. Expert Ver Anti Infect ther. 13(9):1047-1050. https://doi.org/10.1586/14787210.2015.1068119

Brasil. (2020). Sobre a doença. https://coronavirus.saude.gov.br/sobre-a-doenca\#o-que-e-covid

Dilli, D. \& Tesoglu, I. (2020). Cuidados perioperatórios ao recém-nascidos com CC na època do COVID-19. Cardiology in the Young, 30 (7): 946-954.

Ercole, F. F., Melo, L. S., \& Alcoforado, C. L. G. C. (2014). Revisão Integrativa versus Revisão Sistemática. Revista Mineira Enfermagem, 18(1), 1-260. http://www.dx.doi.org/10.5935/1415-2762.20140001

Freitas, B. H. B.Martins, Alves, M. D. S. M. \& Gaíva, M. A. M. (2020). Medidas de prevenção e controle de infecção neonatal por COVID-19: revisão de escopo. Revista Brasileira de Enfermagem, 73(2), 1-10. https://doi.org/10.1590/0034-7167-2020-0467

Góes, F. G.B, Santos, A. S. T, Lucchese, I, Silva, L. J, Silva, L. F. \& Silva, M. A. (2020). Boas práticas no cuidado ao recém-nascido em tempos de covid-19: revisão integrativa. Texto \& Contexto Enfermagem, 29,1-17. https://doi.org/10.1590/1980-265x-tce-2020-0242

Ledo, B. C., Góes, F. G. B., Santos, A. S. T, Pereira-Ávila, F. M. V., Silva, A. C. S. S., \& Bastos, M. P. C. (2021). Fatores associados às práticas assistenciais ao recém-nascido na sala de parto. Escola Anna Nery, 25(1). https://dx.doi.org/10.1590/2177-9465-ean-2020-0102

Li, F., Feng, Z. C. \& Shi, Y. (2020). Proposal for prevention and control of the 2019 novel coronavirus disease in newborn infants. Archives of Disease in Childhood - Fetal and Neonatal Edition. 105(6), 683-684. http://dx.doi.org/10.1136/archdischild-2020-318996

Mascarenhas, V. H. A., Caroci-Becker, A., Venâncio, K. C. M. P., Baraldi, N. G., Durkin, A. C., \& Riesco, M. L. G. (2020). Recomendações assistenciais à parturiente, puérpera e recém-nascido durante a pandemia de COVID-19: revisão de escopo. Rev. Latino-Am. Enfermagem, 28,1-12. http://dx.doi.org/10.1590/1518-8345.4596.3359

OPAS. Pan American Health Organization (2020,09/09). Covid-19 pode reverter décadas de progresso em direção à eliminação de mortes infantis evitáveis alertam agencias. https://www.paho.org/bra/index.php?option=com_content \&view=article\&id=6274:covid-19-pode-reverter-decadas-de-progresso-emdirecao-a-eliminacao-de-mortes-infantis-evitaveis-alertam-agencias \&Itemid= 820

Procianoy, R. S., Silveira, R. C., Manzoni, P., \& Sant'Anna, G. (2020). Neonatal COVID-19: little evidence and the need for more information. Jornal de Pediatria, 96(3), 269-272. https://doi.org/10.1016/j.jped.2020.04.002

Pereira, A. S., Shitsuka, D. M., Parreira, F. J., \& Shitsuka, R. (2018). Metodologia da pesquisa científica. UFSM.

Rose, D. U., Piersigill, F., Ronchetti, M. P., Santisi, A., Bersani, Iliana., Dotta, Andrea., Danhaive, O., \& Auriti. C. (2020). Novel Coronavirus disease (COVID-19) in newborns and infants: what we know so far. Italian Journal of pediatrics, 46(56). https://ijponline.biomedcentral.com/articles/10.1186/s13052-020-0820-x

Sociedade Brasileira de Pediatria. (2020). Recomendações para Assistência ao Recém-Nascido na sala de parto de mãe com COVID-19 suspeita ou confirmada. Atualização 2. https://www.sbp.com.br/fileadmin/user_upload/22499c-NA-Assist_RN_SalaParto_de_mae_com_COVID-19.pdf

Virani, A. K., Puls, H. T., Mitsos, R., Longstaff, H., Goldman, R. D., \& Lantos, J. D. (2020). Benefits and Risks of Visitor Restrictions for Hospitalized Children During the COVID Pandemic. Pediatrics. 146(2). https://doi.org/10.1542/peds.2020-000786

Wang, L., Shi, Y., Xiao, T., Fu, J., Feng, X., Mu, D., Feng, Q., Hei, M., Hu, X., Li, Z., Lu, G., Tang, Z., Wang, Y., Wang, C., Xia, S., Xu, J., Yang, Y., Yang, J., Zeng, M., Zheng, J., Zhou, W., Zhou, X., Zhou, X., Du, L., Lee, S. K., \& Zhou, W. (2020). Consenso de especialistas chineses sobre o manejo perinatal e neonatal para a prevenção e controle da nova infecção por coronavírus em 2019 (primeira edição). Annals of Translational Medicine, 8(3): 47. $10.21037 /$ atm.2020.02.20

World Health Organization (2020). Infection prevention and control during health care when COVID-19 is suspected. https://www.who.int/publications/i/item/10665-331495 